\title{
The silent base flow and the sound sources in a laminar jet
}

\author{
Samuel Sinayoko ${ }^{\text {a) }}$ \\ Institute of Sound and Vibration Research, University of Southampton, Southampton SO17 IBJ, \\ United Kingdom

\begin{abstract}
Anurag Agarwal
Department of Engineering, University of Cambridge, Cambridge CB2 1BZ, United Kingdom
\end{abstract}

(Received 28 April 2011; revised 6 December 2011; accepted 6 December 2011)

\begin{abstract}
An algorithm to compute the silent base flow sources of sound in a jet is introduced. The algorithm is based on spatiotemporal filtering of the flow field and is applicable to multifrequency sources. It is applied to an axisymmetric laminar jet and the resulting sources are validated successfully. The sources are compared to those obtained from two classical acoustic analogies, based on quiescent and time-averaged base flows. The comparison demonstrates how the silent base flow sources shed light on the sound generation process. It is shown that the dominant source mechanism in the axisymmetric laminar jet is "shear-noise," which is a linear mechanism. The algorithm presented here could be applied to fully turbulent flows to understand the aerodynamic noise-generation mechanism.
\end{abstract}

(C) 2012 Acoustical Society of America. [DOI: 10.1121/1.3672804]

PACS number(s): 43.28.Ra, 43.60.Gk, 43.20.Wd [AH]

Pages: $1959-1968$

\section{INTRODUCTION}

Jet noise is one of the dominant aircraft engine sound sources, particularly during takeoff. It remains difficult to reduce because of a lack of understanding of the physical mechanism of noise generation. A key problem is that noise sources get dominated by the much larger hydrodynamic fluctuations and become inaccessible.

Equivalent sound sources can however be computed by means of an acoustic analogy: the sound field generated by these sources is identical to the sound radiating in the far field within the original flow. ${ }^{1-3}$ However, two different noise sources can generate the same sound field. It is therefore problematic to base our understanding of noise generation on these equivalent sound sources: the sound producing mechanism for the equivalent sound sources may be completely different than the one occurring in the physical flow field.

In an acoustic analogy, the flow is linearized around a particular base flow, and the associated sound sources are computed. These sources radiate sound within the chosen base flow, not the physical flow. Possible choices for the base flow include a quiescent medium, ${ }^{2}$ a parallel flow, ${ }^{3}$ and a time-averaged base flow. ${ }^{1,4}$ These base flows are all significantly different from the actual jet, therefore the sound sources are bound to be contaminated by propagation effects and hydrodynamic-field sources.

Goldstein $^{5}$ proposed an alternative approach, in which the base flow corresponds to the nonradiating part of the flow field. Because the radiating part of the flow field is small, the base flow is very close to the real flow. The base flow is silent and we call the associated sounds sources the silent base flow (SBF) sound sources. A key feature of the

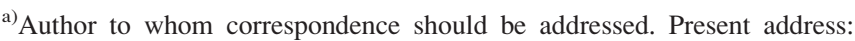
Department of Engineering, University of Cambridge, Cambridge CB2 1BZ, United Kingdom. Electronic mail: s.sinayoko@soton.ac.uk
}

SBF sources is that they are purely radiating: they do not generate hydrodynamic fluctuations.

Although the silent base flow sources generate only sound, the physical sound sources contain and generate both radiating and nonradiating fluctuations. The silent base flow sources should therefore be regarded as the core radiating part of the physical sound sources.

An alternative approach to access the core radiating part of the physical sound sources was used by Cabana et al. ${ }^{6}$ They split Lighthill's source term into ten constituent subterms, and computed the sound generated by each of those terms. The idea was to identify the terms responsible for most of the sound generation. The difficulty of this approach is that each term contains nonradiating components which mask the underlying sound generation mechanisms. Thus, the authors noted that "there is a fine line between source structures which are entirely redundant, and those that are superefficient." The technique presented in this paper uncovers the "superefficient" components present in the flow.

Building on Goldstein's work, Sinayoko et al. ${ }^{7}$ presented a simplified expression for the SBF sources of sound. They showed that it is possible to separate the radiating components from the nonradiating components in a laminar jet, and that the sound sources can be computed.

Using this new approach can help answer some outstanding questions on noise generation in subsonic jets: ${ }^{8}$ what role, if any, do large coherent structures play in jet noise? How are the noise sources distributed in space and time? Answering those questions could lead to innovative strategies for controlling jet noise. The technique could also be further developed to account for the presence of solid boundaries. This would be of great interest to improve our understanding of how solid surfaces give rise to sound sources and affect the sound generated by nearby turbulence.

The flows of practical interest are usually turbulent and involve a broad range of frequencies. However, the algorithm proposed by Sinayoko et al. ${ }^{7}$ employed only spatial filtering, 
which is sufficient only when acoustic radiation is dominated by a single frequency. For a turbulent flow, spatiotemporal filters are required. Understanding how to do this efficiently is crucial for computing the SBF sources in a general flow. In this paper, we address this issue by presenting an algorithm for computing the SBF sources at multiple frequencies. The sources are computed for a flow field obtained from a direct numerical simulation of the Navier-Stokes equations. They are validated by propagating the sound to the far field and by comparing it to the original sound field.

The SBF sources are compared to the sources based on a quiescent base flow (QBF) and a time-averaged base flow (TABF), which correspond respectively to Lighthill's anal$\mathrm{ogy}^{2}$ and Goldstein's analogy. ${ }^{1}$ We show that the silent base flow sources improve our physical understanding beyond what can be achieved from classical acoustic analogies. Finally, we analyze the sources to uncover the dominant noise generation process.

\section{THEORY}

We assume an unbounded, homentropic, perfect gas jet flow of a low Reynolds number $\left(<10^{4}\right.$ based on jet diameter and exit velocity), that is surrounded by a quiescent medium.

Although restrictive, these conditions are sufficient to capture the sound generation from large scale structures in subsonic jets. ${ }^{9}$ Other effects due, for example, to the presence of solid boundaries, ${ }^{10}$ temperature gradients ${ }^{11}$ or supersonic speeds ${ }^{12}$ will be ignored for simplicity.

The flow satisfies the following equations: ${ }^{7}$

$$
\begin{aligned}
& \frac{\partial \rho}{\partial t}+\frac{\partial}{\partial x_{j}} \rho v_{j}=0 \\
& \frac{\partial}{\partial t} \rho v_{i}+\frac{\partial}{\partial x_{j}} \rho v_{i} v_{j}+\frac{\partial}{\partial x_{i}} \pi^{\gamma}=\frac{\partial \sigma_{i j}}{\partial x_{j}}, \\
& \frac{\partial \pi}{\partial t}+\frac{\partial}{\partial x_{j}} \pi v_{j}=0
\end{aligned}
$$

where $\rho$ denotes the density, $\mathbf{v}$ the velocity field, $\sigma_{i j}$ the viscous stress tensor, $\pi=p^{1 / \gamma}$ the modified pressure field, where $p$ is the pressure field, and $\gamma$ the specific heat ratio. In this paper, the viscous terms are assumed to be an insignificant source of sound and will be ignored in the derivation of the sound sources.

\section{A. Silent base flow (SBF) sources}

Using $\mathbf{q}=\{\rho, \rho \mathbf{v}, \pi\}$ as the set of dependent variables, each flow variable $q$ can be decomposed as

$$
q=\bar{q}+q^{\prime},
$$

where $\bar{q}$ denotes the nonradiating part of $q$ and $q^{\prime}$ the radiating part. The radiating part corresponds to the acoustic part of $q$ in the far field. The decomposition is carried out in the frequency-wavenumber domain by observing that, for each frequency $\omega$, the radiating components $q^{\prime}$ lie on the radiating sphere of radius $|\mathbf{k}|=|\omega| / c_{\infty}$ in the wavenumber domain. ${ }^{5,7,13}$ Thus, if $Q$ denotes the space-time Fourier transform of $q$, then

$$
\begin{array}{ll}
\bar{Q}(\mathbf{k}, \omega)=0 \quad \text { if } & |\mathbf{k}|=|\omega| / c_{\infty} \\
\bar{Q}(\mathbf{k}, \omega)=1 \quad \text { if } & |\mathbf{k}| \neq|\omega| / c_{\infty} .
\end{array}
$$

From Sinayoko et al., ${ }^{7}$ the radiating components $\mathbf{q}^{\prime}$ satisfy an equation of the form

$$
\mathbf{L}_{1}\left(\mathbf{q}^{\prime}\right)=\mathbf{s}_{1}
$$

where $\mathbf{L}_{1}$ is a linear operator, $\mathbf{s}_{1}=\left\{0, \mathbf{f}_{1}, 0\right\}$, and $\mathbf{f}_{1}$ represents the momentum equation source term defined as

$$
f_{1 i}=\frac{\partial}{\partial x_{j}}\left(\bar{\rho} \tilde{v}_{i} \tilde{v}_{j}\right)^{\prime}
$$

In Eq. (8), $\tilde{v}_{i}=\overline{\rho v_{i}} / \bar{\rho}$ denotes Favre-averaged velocity. The SBF source $s_{1}$ depends only on the nonradiating flow variables $\overline{\mathbf{q}}$. The full system of equations is given in Appendix A 1.

\section{B. Time averaged base flow (TABF) sources}

If each flow variable $q$ is decomposed as

$$
q=q_{0}+q^{\prime \prime}
$$

where $q_{0}$ denotes the time average of $q$, and $q^{\prime \prime}$ the unsteady fluctuations, the time-averaged base flow can be written as $\mathbf{q}_{0}=\left\{\rho_{0}, \hat{\mathbf{v}}, \pi_{0}\right\}$, where $\hat{\mathbf{v}}=(\rho \boldsymbol{v})_{0} / \rho_{0}$ denotes Favre-averaged mean velocity.

The fluctuating components $\mathbf{q}^{\prime \prime}$ satisfy an equation of the form ${ }^{4,5,7}$

$$
\mathbf{L}_{2}\left(\mathbf{q}^{\prime \prime}\right)=\mathbf{s}_{\mathbf{2}}
$$

where $\mathbf{L}_{2}$ is a linear operator, $\mathbf{s}_{\mathbf{2}}=\left\{0, \mathbf{f}_{2}, 0\right\}$, and $\mathbf{f}_{2}$ represents the momentum equation source term defined as

$$
f_{2 i}=\frac{\partial}{\partial x_{j}}\left(\rho_{0} v_{i}^{\prime \prime} v_{j}^{\prime \prime}\right)^{\prime \prime}-\frac{1}{2} \gamma(\gamma-1) \frac{\partial}{\partial x_{i}}\left(\pi_{0}^{\gamma-2}\left(\pi^{\prime \prime}\right)^{2}\right)^{\prime \prime}
$$

The TABF source $\mathbf{s}_{\mathbf{2}}$ is a function of $\mathbf{q}_{0}$ and $\mathbf{q}^{\prime \prime}$. Since $q^{\prime}$ is embedded in $q^{\prime \prime}, \mathbf{s}_{\mathbf{2}}$ depends on the radiating fluctuations it aims to predict. The full system of equations is given in Appendix A 2.

\section{Quiescent base flow (QBF) sources}

The quiescent base flow $\mathbf{q}_{\infty}$ is defined as

$$
\mathbf{q}_{\infty}=\left\{\rho_{\infty}, \mathbf{0}, \pi_{\infty}\right\}
$$

where $\rho_{\infty}$ and $\pi_{\infty}$ denote, respectively, the uniform density and modified pressure in the far field. The residual components $\mathbf{q}-\mathbf{q}_{\infty}$ satisfy an equation of the form

$$
\mathbf{L}_{3}\left(\mathbf{q}-\mathbf{q}_{\infty}\right)=\mathbf{s}_{3}
$$

where $\mathbf{L}_{3}$ is a linear operator, $\mathbf{s}_{\mathbf{3}}=\left\{0, \mathbf{f}_{3}, 0\right\}$, and $\mathbf{f}_{3}$ is given by 


$$
f_{3 i} \equiv-\frac{\partial}{\partial x_{j}}\left(\rho_{\infty} v_{i} v_{j}\right)-\frac{1}{2} \gamma(\gamma-1) \pi_{\infty}^{\gamma-2} \frac{\partial}{\partial x_{i}}\left(\pi-\pi_{\infty}\right)^{2} .
$$

The above equations have been obtained by substituting $q-q_{\infty}$ for $q^{\prime \prime}$ and $q_{\infty}$ for $q_{0}$ in Eq. (11), for all $q$ in $\{\rho, \rho u$, $\rho v, \pi\}$. The full system of equation is given in Appendix A 3 . Note that if $p$ is used instead of $\pi$ as the dependent variable for pressure, then the gradient term vanishes in Eq. (14). Moreover, because we assume homentropy, there is no source term of the form $p-c_{\infty}^{2} \rho$ (see Ref. 2). The QBF sources, like the TABF sources, depend on the radiating fluctuations they aim to predict. This is not the case for the SBF sources.

\section{ALGORITHM FOR THE SBF DECOMPOSITION}

The expression of Eq. (8) for the SBF sources requires a decomposition of the flow variables into radiating and nonradiating components. An algorithm has been proposed in Sinayoko et al. ${ }^{7}$ for a single-frequency radiating field. Here we derive a more general algorithm, applicable to radiating flow fields composed of multiple frequencies. It shows how to carry out the SBF decomposition efficiently for a general flow field.

For a flow variable $q$, the non-radiating field $\bar{q}$ is computed as follows.

(1) Identify the discrete frequencies $\omega_{1}, \omega_{2}, \ldots \omega_{N}$ which dominate the far field by examining the power spectrum density of $q$ in the far field.

(2) For each radiating frequency $\omega_{i}$, compute $q_{\omega_{i}}(\mathbf{x}, t)$, which represents the instantaneous contribution of frequencies $\pm \omega_{i}$ to $q(\mathbf{x}, t)$ and is defined as

$q_{\omega_{i}}(\mathbf{x}, t)=\frac{1}{2 \pi}\left[Q\left(\mathbf{x}, \omega_{i}\right) e^{-j \omega_{i} t}+Q\left(\mathbf{x},-\omega_{i}\right) e^{j \omega_{i} t}\right]$,

where $Q(\mathbf{x}, \omega)$ is the Fourier transform in time of $q(\mathbf{x}, t)$ :

$Q(\mathbf{x}, \omega)=\int_{-\infty}^{+\infty} q(\mathbf{x}, t) e^{j \omega t} d t$.

$Q\left(\mathbf{x}, \omega_{i}\right)$ can be obtained, for example, using Goertzel's algorithm. ${ }^{14}$ Since, $q(\mathbf{x}, t)$ is real, $Q\left(\mathbf{x},-\omega_{i}\right)$ is the complex conjugate of $Q\left(\mathbf{x}, \omega_{i}\right)$ so Eq. (15) can be written

$$
\begin{aligned}
q_{\omega_{i}}(\mathbf{x}, t)= & \frac{1}{\pi}\left[Q^{R}\left(\mathbf{x}, \omega_{i}\right) \cos \left(\omega_{i} t\right)\right. \\
& \left.+Q^{I}\left(\mathbf{x}, \omega_{i}\right) \sin \left(\omega_{i} t\right)\right],
\end{aligned}
$$

where the $R$ and $I$ superscripts denote the real and imaginary parts. Equation (18) allows us to compute $q_{\omega_{i}}(\mathbf{x}, t)$, for any time $t$, from the real and imaginary parts of $Q\left(\mathbf{x}, \omega_{i}\right)$.

(3) For a given time $t$, for each value of $\omega_{i}$, compute the radiating part $q_{\omega_{i}}^{\prime}(\mathbf{x}, t)$ of $q_{\omega_{i}}(\mathbf{x}, t)$ by using the following procedure:

(a) compute the Fourier transform in space of $Q^{R}\left(\mathbf{x}, \omega_{i}\right)$, denoted $\mathcal{Q}\left(\mathbf{k}, \omega_{i}\right)$,

(b) multiply $\mathcal{Q}\left(\mathbf{k}, \omega_{i}\right)$ by a filter window $\mathcal{W}\left(\mathbf{k}, \omega_{i}\right)$ designed to return the radiating components satisfying $|\mathbf{k}|=\omega_{i} / c_{\infty},{ }^{7}$ which gives $\mathcal{Q}^{\prime}\left(\mathbf{k}, \omega_{i}\right)$, (c) compute the real part of the inverse Fourier transform in space of $\mathcal{Q}^{\prime}\left(\mathbf{k}, \omega_{i}\right)$, which we denote $Q^{R^{\prime}}\left(\mathbf{x}, \omega_{i}\right)$,

(d) repeat the above 3 steps with $Q^{I}\left(\mathbf{x}, \omega_{i}\right)$ instead of $Q^{R}\left(\mathbf{x}, \omega_{i}\right)$ to get $Q^{I^{\prime}}\left(\mathbf{x}, \omega_{i}\right)$,

(e) compute $q_{\omega_{i}}^{\prime}(\mathbf{x}, t)$, by observing that

$$
\begin{aligned}
q_{\omega_{i}}^{\prime}(\mathbf{x}, t)= & \frac{1}{\pi}\left[Q^{R^{\prime}}\left(\mathbf{x}, \omega_{i}\right) \cos \left(\omega_{i} t\right)\right. \\
& \left.+Q^{I^{\prime}}\left(\mathbf{x}, \omega_{i}\right) \sin \left(\omega_{i} t\right)\right] .
\end{aligned}
$$

(4) Compute the radiating part $q^{\prime}(\mathbf{x}, t)$ as

$$
q^{\prime}(\mathbf{x}, t)=\sum_{i=1}^{N} q_{\omega_{i}}^{\prime}(\mathbf{x}, t) .
$$

(5) Compute the nonradiating part $\bar{q}(\mathbf{x}, t)$ as

$$
\bar{q}(\mathbf{x}, t)=q(\mathbf{x}, t)-q^{\prime}(\mathbf{x}, t) .
$$

In practice the signals are discretized and the spatial Fourier transform is carried out using the fast Fourier transform (FFT) algorithm. To avoid aliasing, the signals should be zero-padded in the space-time domain before computing the FFT and filtering the wavenumber-frequency domain.

In the algorithm, step 3 greatly reduces the computational cost of the flow decomposition, in terms of both processing time and memory requirements. To obtain $\bar{q}(\mathbf{x}, t)$, it only is necessary to compute the radiating part of $Q\left(\mathbf{x}, \omega_{i}\right)$ for the dominant radiating frequencies $\omega_{i}$. This is cheaper than computing the full Fourier transform in time and space of $q(\mathbf{x}, t)$ and applying a filter defined over all frequencies and wavenumbers.

\section{SOURCE COMPUTATION IN AN EXCITED AXISYMMETRIC JET}

\section{A. Numerical simulation}

We now consider a nonlinear problem in which an axisymmetric jet is excited by two discrete-frequency axisymmetric disturbances at the jet exit. The frequencies are chosen to trigger some instability waves in the flow. These instability waves grow downstream and interact nonlinearly, generating acoustic waves. The Mach number of the jet is 0.9 and the Reynolds number is 3600 . The jet operating conditions and the base mean flow are chosen to match the experimental data of Stromberg. ${ }^{15}$

Suponitsky et al. ${ }^{16}$ performed direct numerical simulations of the compressible Navier-Stokes equations for this problem. In their simulations the mean flow was prescribed by imposing time-independent forcing terms. They ran simulations with different combinations of excitation frequencies and amplitudes. The data used here corresponds to the combination with the largest acoustic radiation. The two excitation frequencies are $\omega_{1}=2.2$ and $\omega_{2}=3.4$. Sound radiates mainly at the difference frequency $\Delta \omega=1.2$. The results presented in this section have been normalized by using the 
jet diameter $D$, jet exit speed $U_{j}$, and the ambient density as the length, velocity, and density scales, respectively.

\section{B. Results}

\section{Silent base flow sources}

a. Flow decomposition. The three frequencies dominating the far field sound are, in decreasing order of importance, 1.2, 1.0, and 2.2. The contribution of other frequencies to the power spectral density (PSD) at point $(z, r)=(40,20)$, for example, located in the far field in a region of strong acoustic radiation, is at least 20 decibels weaker than the contribution of the peak frequency (1.2). Hence it is sufficient to concentrate on the radiating frequencies $1.0,1.2$, and 2.2 to compute the radiating sound.

We use a Gaussian filter defined in the frequencywavenumber domain as

$$
\mathcal{W}(\mathbf{k}, \omega)=\exp \left[-\frac{\left(|\mathbf{k}|-|\omega| / c_{\infty}\right)^{2}}{2 \sigma^{2}}\right] .
$$

We want the width of the Gaussian, controlled by $\sigma$, to be small to separate the radiating components from the nonra- diating components. However, in the physical domain, the filter is a Gaussian of standard deviation $1 / \sigma$; as $\sigma$ tends to zero, the filter width increases in the physical domain which leads to inaccuracies near the boundaries of the domain. Thus, there is a trade-off between the filter widths in the spectral and physical domains. Here, we have chosen $\sigma=0.37$ after careful examination of the filtered flow fields in the two domains.

The algorithm of Sec. III is applied to the density field $\rho$. Results are shown in Fig. 1. The first row [Figs. 1(a)-1(c)] presents snapshots of $\rho_{1.0}, \bar{\rho}_{1.0}$, and $\rho_{1.0}^{\prime}$, i.e., the SBF decomposition of the density field at frequency 1.0. Similarly, the second and third rows, respectively, present similar decompositions at frequencies 1.2 and 2.2. The last row presents the full density field $\rho$ followed by $\bar{\rho}$ and $\rho^{\prime}$.

The decomposition is successful: the second column of Fig. 1, which contains the nonradiating signals, exhibits flow fields that are silent (no sound is propagating to the far field). On the contrary, in the third column, which contains only radiating components, the flow fields are acoustic in nature and contain no hydrodynamic fluctuations.

The decomposition is superior to the one presented by Sinayoko et al., ${ }^{7}$ in which the flow field was filtered in the wavenumber domain only by focusing on the dominant

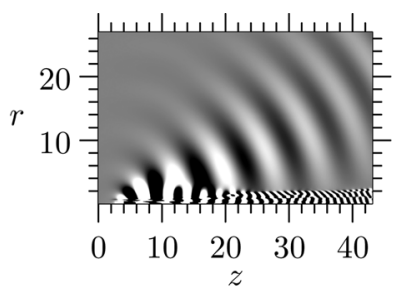

(a) $\rho_{1.0}$

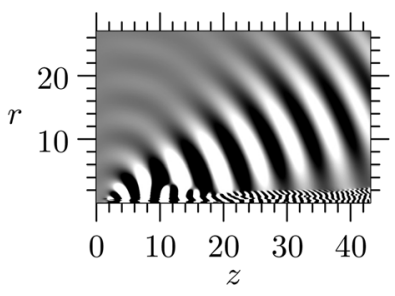

(d) $\rho_{1.2}$

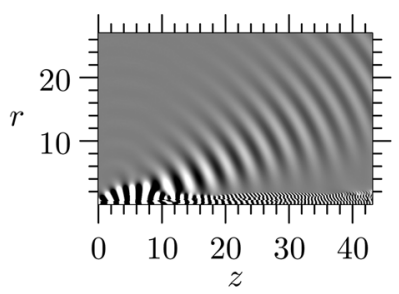

(g) $\rho_{2.2}$

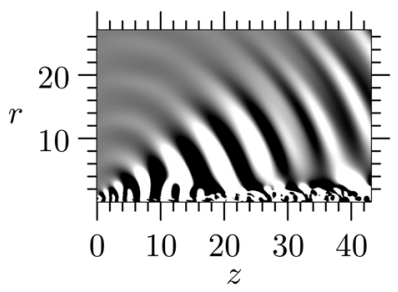

(j) $\rho-\rho_{0}$

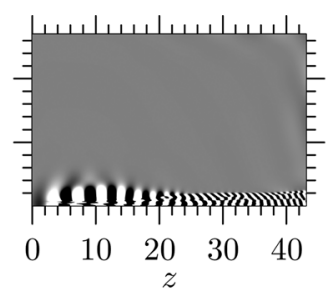

(b) $\bar{\rho}_{1.0}$

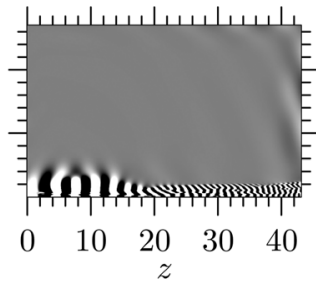

(e) $\bar{\rho}_{1.2}$

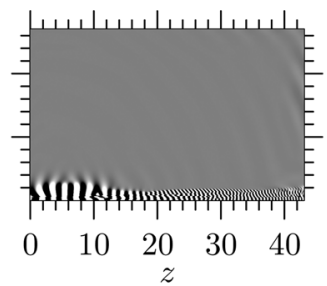

(h) $\bar{\rho}_{2.2}$

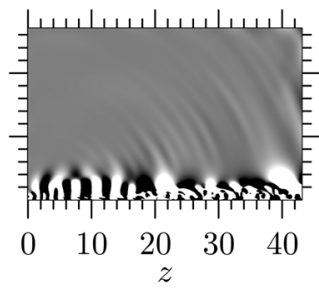

(k) $\bar{\rho}-\rho_{0}$

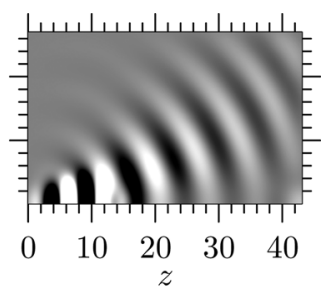

(c) $\rho_{1.0}^{\prime}$

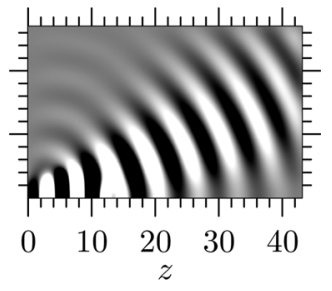

(f) $\rho_{1.2}^{\prime}$

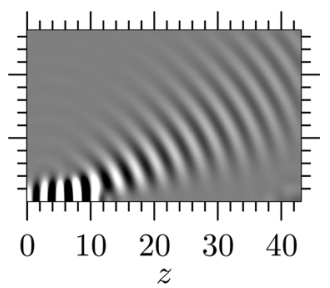

(i) $\rho_{2.2}^{\prime}$

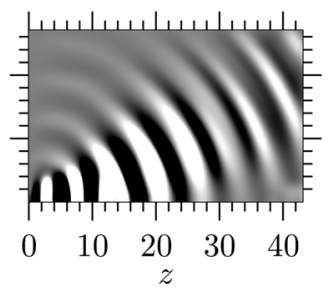

(1) $\rho^{\prime}$
FIG. 1. Decomposition of the density field $\rho$ into non-radiating components $\bar{\rho}$ and radiating components $\rho^{\prime}$. The field $\rho_{\omega_{i}}$, where $\omega_{i} \in\{1.0,1.2,2.2\}$, denotes snapshots of the density field at the radiating frequencies. The color scales are from $-2 \times 10^{-5}$ to $2 \times 10^{-5}$. 
radiating frequency $\omega=1.2$. The proposed algorithm offers an efficient way of decomposing a general flow field containing a broad range of frequencies into its nonradiating and radiating components.

b. SBF sources. We present the SBF sources at frequency 1.2. Similar results have been obtained for the other two radiating frequencies, 1.0 and 2.2. From Eq. (8), the computation of the axial and radial SBF sources requires a second filtering step that is applied to $-\partial\left(\bar{\rho} \tilde{\mathbf{v}} \tilde{v}_{j}\right) / \partial x_{j}$. This filtering operation has been carried out by means of a Gaussian filter of standard deviation $\sigma$, defined as

$$
\sigma(\omega)=\frac{1}{7.5} \frac{\omega}{c_{\infty}} .
$$

The above equations can be expressed as $\sigma \approx 1 / 1.2 \lambda$, where $\lambda=2 \pi c_{\infty} / \omega$ denotes the acoustic wavelength. Therefore, in the physical domain, the standard deviation of the filter $(1 / \sigma)$ is about one acoustic wavelength.

The filter width defined above for the source computation is different from the constant width $\sigma=0.37$ used for the flow decomposition of Fig. 1. The latter value is, respectively, 3, 2.5, and 1.4 times larger than the width of the filter used for the source computation at frequencies 1.0, 1.2, and 2.2. Thus the filter used for the flow decomposition is wider in the spectral domain. Using a wider filter is possible because of the clear separation between the radiating and nonradiating components. There is however no clear separation between the radiating and nonradiating components in the Fourier transform of the term $-\partial\left(\bar{\rho} \tilde{\mathbf{v}} \tilde{v}_{j}\right) / \partial x_{j}$, and the value of $\sigma$ therefore controls the energy of the SBF sources. There is no simple way of guessing the right value of $\sigma$ for the source computation. As will be shown later, Eq. (22), which has been defined empirically, leads to satisfactory results for the present flow. It remains to be investigated if this equation is applicable to more general flows. Nevertheless, we have observed that the structure of the SBF sources remains the same for a broad range of values of $\sigma$. The physical behavior of the SBF sources is therefore relatively insensitive to the value of $\sigma$.

Since the flow is axisymmetric, any vector variable $\mathbf{q}$ is expressed in terms of an axial component $q_{z}$ and a radial component $q_{r}$. The divergence of $\mathbf{q}$ is given by

$$
\frac{\partial q_{j}}{\partial x_{j}}=\frac{1}{r} \frac{\partial\left(r q_{r}\right)}{\partial r}+\frac{\partial q_{z}}{\partial z} .
$$

Note also that the three-dimensional Fourier transform of an axisymmetric scalar variable $q(r, z)$ is given by its Hankel transform of order 0 in the radial direction, combined with its Fourier transform in the axial direction. To compute the three dimensional Fourier transform of a vector $\mathbf{q}$ expressed as $\left(q_{r}, q_{z}\right)$, one can use the above technique for the axial component $q_{z}$. For the radial component $q_{r}$, one should use a Hankel transform of order 1 instead of a Hankel transform of order 0 (see Appendix B).

Snapshots of $\left(f_{1 z}\right)_{1.2}$ and $\left(f_{1 r}\right)_{1.2}$, the momentumequations SBF sources at frequency 1.2, are presented in
Figs. 2(a) and 2(b), respectively. The axial SBF source $f_{1 z}$ takes the form of a wave-packet centered around the jet axis. The envelope of the wavepacket reaches a maximum at $z=5$, which corresponds to the end of the potential core, and becomes negligible for $z \geq 20$. The radial SBF source $f_{1 r}$ is concentrated outside the jet but close to the jet axis, around $r=2$. It is negligible for $z \geq 5$. Despite the different flow decomposition algorithm, these results are consistent with the ones presented by Sinayoko et al. ${ }^{7}$

Although one might expect the sound sources to be distributed within the jet region, the SBF sources extend beyond the jet region in the radial direction. The sources of sound depend on the hydrodynamic fluctuations, which extend several jet diameters in the radial direction. It is hence possible that the sound sources are distributed within the near field of the jet.

c. Validation. To validate the SBF sources, we propagate the sound to the far field by solving the Euler Equations linearized about the time averaged base flow. These equations do not use the linear operator $\mathbf{L}_{1}$ on the left hand side of Eq. (7), but they should be a good approximation for it, as explained by Sinayoko et al. ${ }^{7}$

The propagated sound is compared to the radiating part of the DNS solution. The results are presented in Fig. 3. The DNS solution 3(a) is in good agreement with the sound radiating from the SBF sources 3(b). A quantitative comparison along $r=20$ [Fig. 3(c)] confirms this. These results suggest that the SBF sources capture the main sound generation mechanism.

\section{TABF sources}

The TABF sources $\left(f_{2 z}\right)_{1.2}$ and $\left(f_{2 r}\right)_{1.2}$, are computed using Eq. (11). The steady flow field $\mathbf{q}_{0}$ is computed by averaging 300 snapshots uniformly distributed over a duration $T=31.4259$ that corresponds to $6 T_{1.2}$, where $T_{1.2}$ is the time period associated with frequency $\omega=1.2$. The results are shown in Fig. 4.

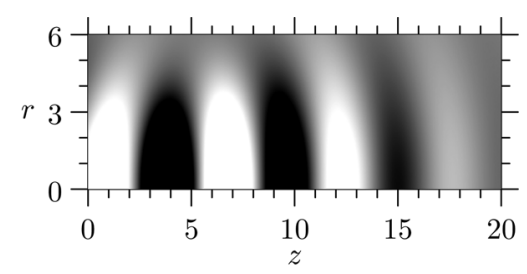

(a) Axial NRBF source $\left(f_{1 z}\right)_{1.2}$.

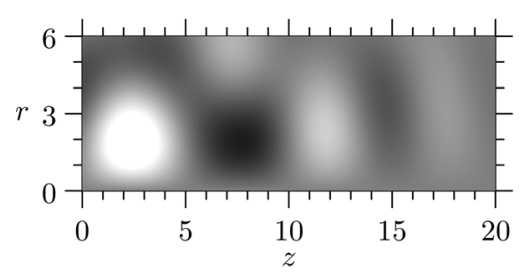

(b) Radial NRBF source $\left(f_{1 r}\right)_{1.2}$

FIG. 2. Snapshots of SBF sources $\left(f_{1 z}\right)_{1.2}$ and $\left(f_{1 r}\right)_{1.2}$ at frequency 1.2. Color scales are from $-2 \times 10^{-6}$ to $2 \times 10^{-6}$. 


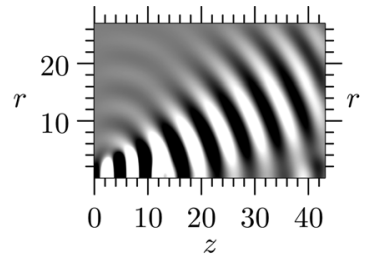

(a) $\rho_{1.2}^{\prime}$ from DNS

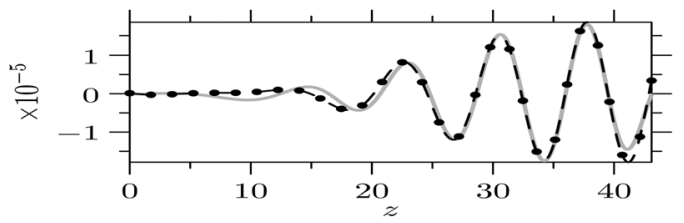

(c) profiles of $\rho-\rho_{0}$ and $\rho_{1.2}^{\prime}$ for $r=20$

FIG. 3. Comparison of (a) the radiating density field $\rho_{1.2}^{\prime}$ with (b) the density field $\rho-\rho_{0}$ obtained by driving the LEE with the SBF sources $\left(f_{1 z}\right)_{1.2}$ and $\left(f_{1 r}\right)_{1.2}$. Color scales are from $-2 \times 10^{-5}$ to $2 \times 10^{-5}$. Figure (c) gives profiles along $r=20$ : the gray line represents the DNS solution $\left(\rho_{1.2}\right)$ and the dashed black line with black circles the LEE solution $\left(\rho-\rho_{0}\right)$.

The structure of the TABF sources is very different from that of the SBF sources: the TABF sources are dominated by higher wavenumbers. These wavenumbers are of the form $\omega / u_{c}$, where $u_{c}<c_{\infty}$ is a convection speed, as opposed to $\omega / c_{\infty}$ for the radiating wavenumber that dominate the SBF sources. The TABF sources are maximum around $z=15$, where the SBF sources are negligible. The amplitude of the TABF sources is three orders of magnitude larger than that of the SBF sources.

The TABF sources $\left(f_{2 z}\right)_{1.2}$ and $\left(f_{2 r}\right)_{1.2}$ are used to drive the linearized Euler equations.

The radiating sound field is compared to that obtained from the DNS solution in Fig. 5. Agreement is reasonable although the noise radiating from the TABF sources is larger than that from the DNS by about $20 \%$. This discrepancy might originate from imperfect noise cancellations between the sound fields radiating from the different terms composing the TABF sources. Note that such cancellations are not occurring for the other sound sources considered in this paper, where most of the sound is originating from just a single term.

\section{QBF sources}

The QBF sources $f_{3 z}$ and $f_{3 r}$, are computed using Eq. (14). The results are shown in Fig. 6. The axial and radial momentum QBF sources exhibit wavepacket structures that are centered respectively around the jet axis and the jet shear layer, and extend approximately between 5 and 18 jet diameters in the axial direction.

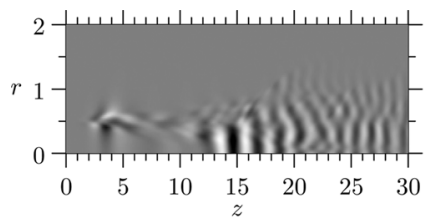

(a) Axial TABF source $\left(f_{2 z}\right)_{1.2}$

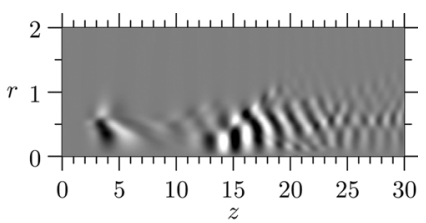

(b) Radial TABF source $\left(f_{2 r}\right)_{1.2}$
FIG. 4. Snapshots of TABF sources $\left(f_{2 z}\right)_{1.2}$ and $\left(f_{2 r}\right)_{1.2}$ at frequency 1.2 . Color scales are from $7.5 \times 10^{-3}$ to $7.5 \times 10^{-3}$.

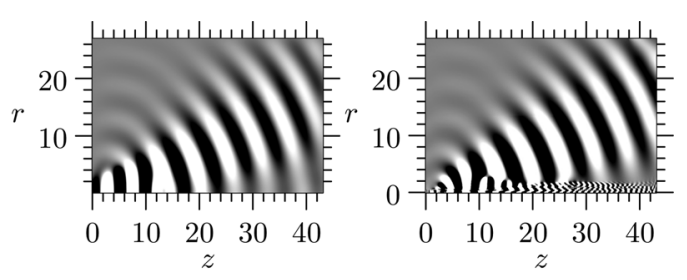

(a) $\rho_{1.2}^{\prime}$ from DNS (b) $\rho-\rho_{0}$ from LEE

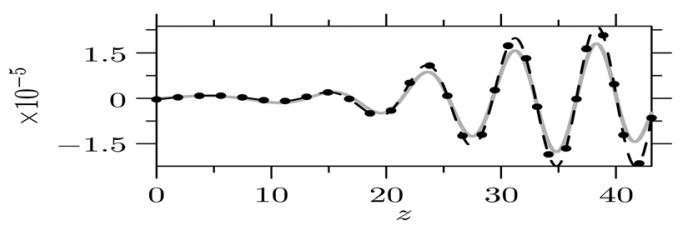

(c) profiles of $\rho-\rho_{0}$ and $\rho_{1.2}^{\prime}$ for $r=20$

FIG. 5. Comparison of (a) the radiating density field $\rho_{1.2}^{\prime}$ with (b) the density field $\rho-\rho_{0}$ obtained by driving the LEE with the TABF sources $\left(f_{2 z}\right)_{1.2}$ and $\left(f_{2 r}\right)_{1.2}$. Color scales are from $-2 \times 10^{-5}$ to $2 \times 10^{-5}$. Figure (c) gives profiles along $r=20$ : the gray line represents the DNS solution $\left(\rho_{1.2}\right)$ and the dashed black line with black circles the LEE solution $\left(\rho-\rho_{0}\right)$.

Compared to the TABF sources of Fig. 4, the QBF sources have a higher amplitude (roughly one order of magnitude), due to the presence of terms that are linear in the unsteady flow variables. The QBF sources $\left(f_{3 z}\right)_{1.2}$ and $\left(f_{3 r}\right)_{1.2}$ are used to drive the linearized Euler equations, with a quiescent mean flow. The radiating sound field is compared to the one obtained from the DNS solution in Fig. 7. The sound radiating from the QBF sources is in very good agreement with the DNS solution. Small differences for $z \geq 35$ are due to the 100 points buffer used as a boundary condition.

Compared to the SBF sources shown in Fig. 2, the QBF sources have an amplitude that is at least 3 orders of magnitude larger. This is due to the non-radiating components that are present in the QBF sources. It makes them difficult to interpret directly as sound sources.

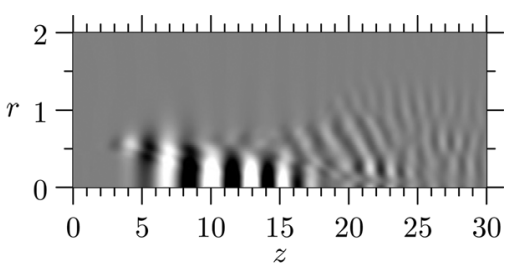

(a) Axial NRBF source $\left(f_{1 z}\right)_{1.2}$.

Color scales are from $-5 \times 10^{-2}$ to $5 \times 10^{-2}$.

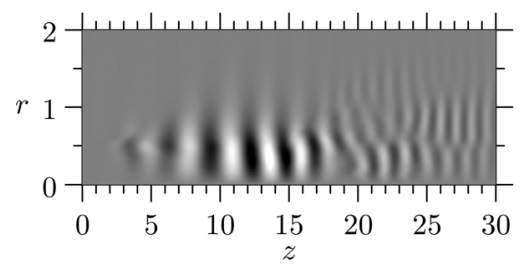

(b) Radial NRBF source $\left(f_{1 r}\right)_{1.2}$. Color scales are from $-3 \times 10^{-2}$ to $3 \times 10^{-2}$.

FIG. 6. Snapshots of SBF sources $\left(f_{1 z}\right)_{1.2}$ and $\left(f_{1 r}\right)_{1.2}$ at frequency 1.2. 

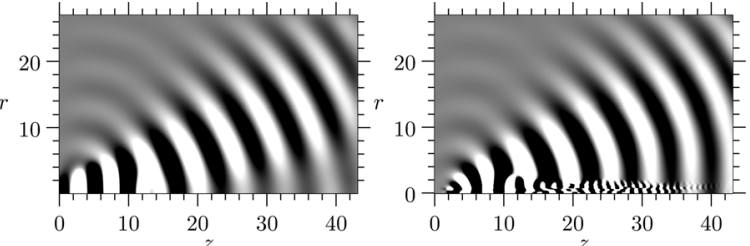

(a) $\rho_{1.2}^{\prime}$ from DNS

(b) $\rho-\rho_{0}$ from LEE

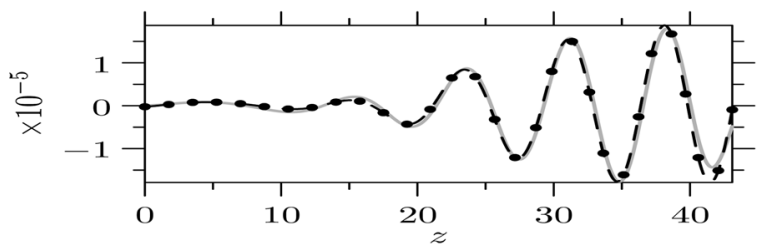

(c) profiles of $\rho-\rho_{0}$ and $\rho_{1.2}^{\prime}$ for $r=20$

FIG. 7. Comparison of (a) the radiating density field $\rho_{1.2}^{\prime}$ with (b) the density field $\rho-\rho_{0}$ obtained by driving the LEE with the QBF sources $\left(f_{3 z}\right)_{1.2}$ and $\left(f_{3 r}\right)_{1.2}$. Color scales are from $-2 \times 10^{-5}$ to $2 \times 10^{-5}$. Figure (c) gives profiles along $r=20$ : the gray line represents the DNS solution $\left(\rho_{1.2}\right)$ and the dashed black line with black circles the LEE solution $\left(\rho-\rho_{0}\right)$.

\section{Filtered QBF sources}

Within the source region, the radiating components are small compared to the nonradiating ones. It can therefore be argued that $\left(\rho v_{i} v_{j}\right)^{\prime} \approx\left(\bar{\rho} \tilde{v}_{i} \tilde{v}_{j}\right)^{\prime}$, and that the SBF sources can be approximated by the radiating part of the QBF sources.

The filtered QBF sources, $\left(f_{3 z}\right)_{1.2}^{\prime}$ and $\left(f_{3 r}\right)_{1.2}^{\prime}$, are computed by applying a Gaussian nonradiating filter of standard deviation $\sigma(1.2)$ [using Eq. (22)]. The results are shown in Fig. 8. The filtered QBF sources exhibit wavepacket structures.

The filtered QBF sources are validated in Fig. 9. The far field sound and the sound radiating from the filtered QBF sources are in good agreement for $r=20$. The fluctuations generated by the filtered QBF sources are purely acoustic: they do not contain any hydrodynamic fluctuations as was the case for the TABF sources (Fig. 5) and the QBF sources (Fig. 7). Note however, that, in the near field, the agreement between the DNS solution and the sound radiating from the sources is poorer for the filtered QBF sources [Figs. 9(a) and 9(b)] than for the SBF sources [Figs. 3(a) and 3(b)].

\section{Discussion}

All the source terms computed above are sufficient to generate the acoustic field that is radiating within the original flow field. However, they have very different structures and amplitudes.

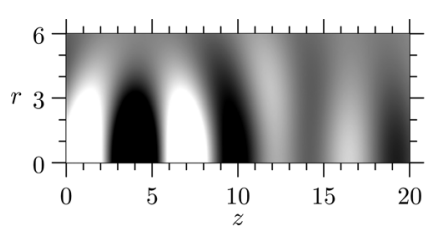

(a) Filtered axial QBF source

$\left(f_{1 z}\right)_{1.2}^{\prime}$. Color scales are from $-8 \times 10^{-5}$ to $8 \times 10^{-5}$.

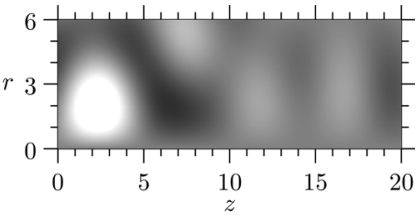

(b) Filtered radial QBF source $\left(f_{1 r}\right)_{1.2}^{\prime}$. Color scales are from $-2 \times 10^{-5}$ to $2 \times 10^{-5}$.
FIG. 8. Snapshots of filtered QBF sources $\left(f_{3 z}\right)^{\prime}{ }_{1.2}$ and $\left(f_{3 r}\right)^{\prime}{ }_{1.2}$ at frequency 1.2 .
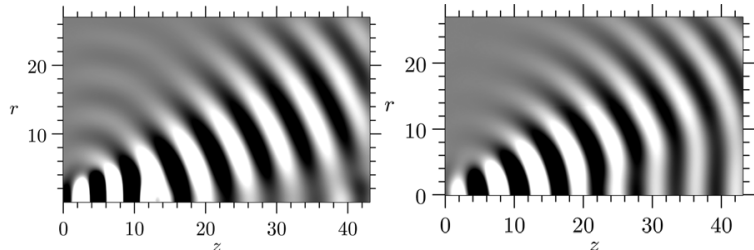

(a) $\rho_{1.2}^{\prime}$ from DNS

(b) $\rho-\rho_{0}$ from LEE

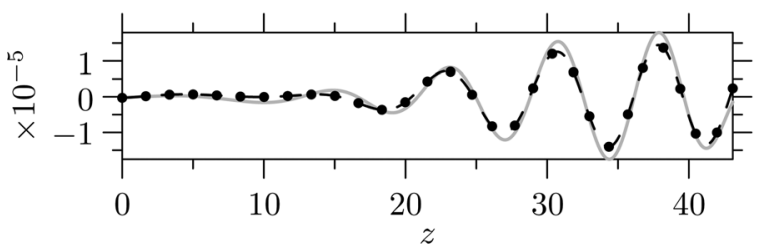

(c) profiles of $\rho-\rho_{0}$ and $\rho_{1.2}^{\prime}$ for $r=20$

FIG. 9. Comparison of (a) the radiating density field $\rho_{1.2}^{\prime}$ with (b) the density field $\rho-\rho_{0}$ obtained by driving the LEE with the radiating QBF sources $\left(f_{3 z}\right)^{\prime}{ }_{1.2}$ and $\left(f_{3 r}\right)^{\prime}{ }_{1.2}$. Color scales are from $-2 \times 10^{-5}$ to $2 \times 10^{-5}$. Figure (c) gives profiles along $r=20$ : the gray line represents the DNS solution $\left(\rho_{1.2}\right)$ and the dashed black line with black circles the LEE solution $\left(\rho-\rho_{0}\right)$.

Compared to the amplitude of the sound field, the TABF and QBF sources have a much larger amplitude. From Figs. 4(a) and 6(a), the axial TABF source $\left(f_{2 z}\right)_{1.2}$ and the axial QBF source $\left(f_{3 z}\right)_{1.2}$ are, respectively, 2 and 3 orders of magnitude larger than the acoustic fluctuations. The SBF and filtered QBF sources have a similar amplitude to that of the sound field.

This discrepancy in amplitude is due to the presence of non-radiating components within the source terms. Most of the components in the TABF and QBF sources are hydrodynamic, whereas the SBF and filtered QBF sources are purely radiating. The TABF and $\mathrm{QBF}$ sources therefore generate significant hydrodynamic fluctuations, which can be seen travelling along the jet axis $(r=0)$ in Figs. 5(b) and 7(b). On the contrary, the SBF and filtered QBF sources do not generate hydrodynamic fluctuations [compare with Figs. 3(b) and 9(b)].

The presence of nonradiating components within the flow has a big impact on the structure of the source terms: the TABF and QBF source terms (Figs. 4 and 6) are completely different from the SBF and filtered QBF source terms (Figs. 2 and 8). More precisely, the TABF and QBF sources are dominated by high (hydrodynamic) wavenumbers, whereas the SBF and QBF sources are dominated by low (acoustic) wavenumbers. It is necessary to remove the nonradiating components from the source terms to uncover the silent base flow sources.

The filtered QBF sources exhibit similar desirable properties as the SBF sources: their amplitudes are of the same order of magnitude as that of the sound field and they generate only sound. However, the SBF sources are different from the filtered QBF sources: the SBF sources are based on the nonradiating base flow whereas the filtered QBF sources are based on the full flow field. It is therefore easier to compute the filtered QBF sources than the SBF sources. It is all the more important to understand the benefits that the SBF sources have to offer compared to the filtered QBF sources. The filtered QBF sources can be decomposed as 


$$
\begin{aligned}
\left(f_{3 i}\right)^{\prime}= & \frac{\partial}{\partial x_{j}}\left(\rho_{\infty} v_{i} v_{j}\right)^{\prime} \\
& -\frac{1}{2} \gamma(\gamma-1) \pi_{\infty}^{\gamma-2} \frac{\partial}{\partial x_{i}}\left[\left(\pi-\pi_{\infty}\right)^{2}\right]^{\prime} \\
\approx & f_{1 i}+\frac{\partial}{\partial x_{j}}\left(\rho_{\infty} \tilde{v}_{j} v_{i}^{\prime}\right)^{\prime}+\frac{\partial}{\partial x_{j}}\left(\rho_{\infty} \tilde{v}_{i} v_{j}^{\prime}\right)^{\prime} .
\end{aligned}
$$

Here we have assumed $\rho_{\infty} \approx \bar{\rho}$ and have neglected the quadratic terms in the radiating variables. The modified pressure term has also been neglected: it is equal to the square of density fluctuations and is not a significant sound source in the present flow. Equation (25) shows that the filtered QBF sources contain some extra terms compared to the SBF sources. These extra terms are linear in the radiating flow field. Comparing Figs. 2(b) and 8(b) shows that these terms have a significant effect. These linear terms have radiating components with an amplitude similar to that of the SBF sources. They explain the differences that can be observed in the near field $(r \leq 10)$ between the sound radiating from the filtered QBF sources [Fig. 9(b)] and the sound radiating from the SBF sources [Fig. 3(b)].

In the above, we have only discussed the source terms themselves. However, the linear operator on the left hand side of the equations governing the propagation of sound can generate further radiating components. These radiating components come, for example, from the interaction of hydrodynamic components with a nonuniform base flow. Thus, the TABF sources generate vorticity waves which are converted into acoustic waves after interacting with the diverging base flow. This makes the TABF sources more complicated to interpret: the sources do not contain all the sound generating terms. On the contrary, the QBF and filtered QBF sources, do not suffer from this problem as they radiate within a uniform flow. Moreover, although the SBF radiate within a nonuniform flow, they also avoid this pitfall because they do not generate hydrodynamic fluctuations.

\section{Source identification}

\section{Decomposition of the SBF sources}

The expression of Eq. (8) can be further decomposed to identify the radiating core of the SBF sources. In an axisymmetric flow, the SBF sources in the momentum equation can be written as

$$
f_{1 z}=f_{1 z z}+f_{1 z r}, \quad f_{1 r}=f_{1 r z}+f_{1 r r},
$$

where

$$
\begin{aligned}
& f_{1 z z}=-\frac{\partial}{\partial z}\left(\bar{\rho} \tilde{v}_{z} \tilde{v}_{z}\right)^{\prime}, \quad f_{1 z r}=-\frac{1}{r} \frac{\partial}{\partial r}\left(r \bar{\rho} \tilde{v}_{z} \tilde{v}_{r}\right)^{\prime}, \\
& f_{1 r z}=-\frac{\partial}{\partial z}\left(\bar{\rho} \tilde{v}_{r} \tilde{v}_{z}\right)^{\prime}, \quad f_{1 r r}=-\frac{1}{r} \frac{\partial}{\partial r}\left(r \bar{\rho} \tilde{v}_{r} \tilde{v}_{r}\right)^{\prime} .
\end{aligned}
$$

The sound fields from these sources are obtained by driving the LEE with them (see Fig. 10). It is clear that the cross

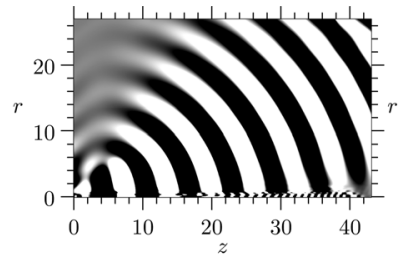

(a)

(c)

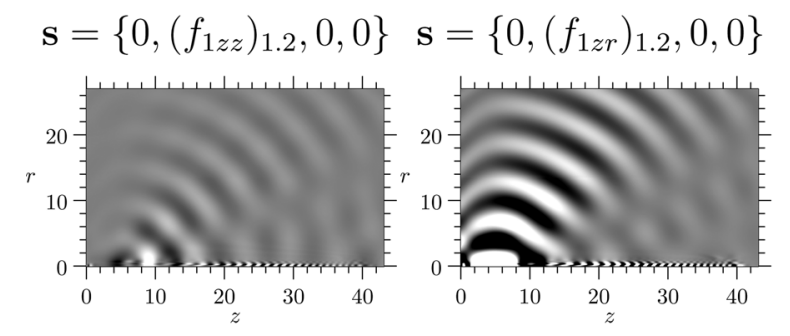

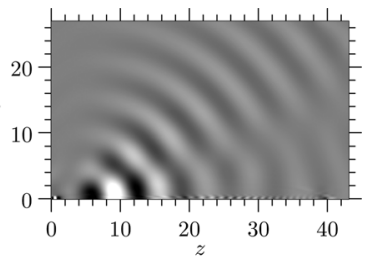

(b) (d)

$$
\mathbf{s}=\left\{0,0,\left(f_{1 r z}\right)_{1.2}, 0\right\} \quad \mathbf{s}=\left\{0,0,\left(f_{1 r r}\right)_{1.2}, 0\right\}
$$

FIG. 10. Snapshots of the modified pressure field radiating from the source terms that compose the SBF sources $\left(f_{1 z}\right)_{1.2}$ and $\left(f_{1 r}\right)_{1.2}$, compared to the DNS solution $\rho_{1.2}$. Color scales are from $-2 \times 10^{-6}$ to $2 \times 10^{-6}$.

terms $f_{1 z r}$ and $f_{1 r z}$ generate negligible sound compared to the terms $f_{1 z z}$ and $f_{1 r r}$. The axial term $f_{1 z z}$ is responsible for most of the sound radiation. However, the radial term $f_{1 r r}$ generates significant sound radiation at large angles to the jet axis. These results are consistent with the findings of Suponitsky et al. ${ }^{17}$

\section{Shear noise}

The term $f_{1 z z}$ can be further decomposed by splitting the nonradiating velocity $\tilde{v}_{z}$ into a mean part $\tilde{v}_{z 0}$ and an hydrodynamic parts $\tilde{v}_{z}^{\prime \prime}$ :

$$
\tilde{v}_{z}=\tilde{v}_{z 0}+\tilde{v}_{z}^{\prime \prime}
$$

Note that $v_{z 0}$ is the time-averaged part of $\tilde{v}_{z}$ and is therefore different from $v_{z 0}$. In the main sound generating region $(z \leq 15, r \leq 0.5)$, we can also assume that $\tilde{v}_{z}^{\prime \prime} \ll \tilde{v}_{z 0}$. If we neglect the effect of density fluctuations, by writing $\bar{\rho}_{\infty} \approx \rho_{\infty}$, then

$$
f_{1 z z} \approx-\rho_{\infty} \frac{\partial}{\partial z}\left(\tilde{v}_{z 0} \tilde{v}_{z 0}+2 \tilde{v}_{z 0} \tilde{v}_{z}^{\prime \prime}+\tilde{v}_{z}^{\prime \prime} \tilde{v}_{z}^{\prime \prime}\right)^{\prime} .
$$

The first term in the brackets is steady so its radiating part is zero. The third term is quadratic in the hydrodynamic part of $v_{z}$ : it can be neglected compared to the second term.

Therefore, $f_{1 z z}$ can be approximated by

$$
f_{1 z z} \approx-2 \rho_{\infty} \frac{\partial}{\partial z}\left(\tilde{v}_{z 0} \tilde{v}_{z}^{\prime \prime}\right)^{\prime} .
$$

The above expression corresponds to radiating part of the "shear noise" term, which has been identified as the dominant source terms in several studies. ${ }^{18,19}$ 

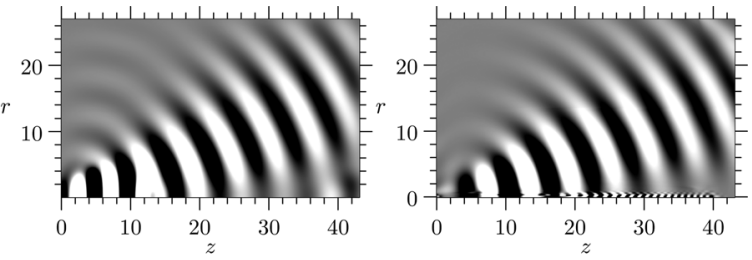

(a) $\rho_{1.2}^{\prime}$ from DNS

(b) $\rho-\rho_{0}$ from LEE

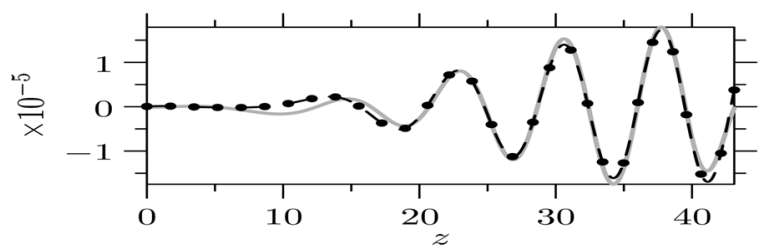

(c) profiles of $\rho-\rho_{0}$ and $\rho_{1.2}^{\prime}$ for $r=20$

FIG. 11. Comparison of (a) the radiating density field $\rho_{1.2}^{\prime}$ with (b) the density field $\rho-\rho_{0}$ obtained by driving the LEE with the shear noise term $\left(f_{1 z z}\right)_{1.2}$ of Eq. (31). Color scales are from $-2 \times 10^{-5}$ to $2 \times 10^{-5}$. Figure (c) gives profiles along $r=20$ : the gray line represents the DNS solution $\left(\rho_{1.2}\right)$ and the dashed black line with black circles the LEE solution $\left(\rho-\rho_{0}\right)$.

The sound radiating from the "shear noise" term of Eq. (31) is shown in Fig. 11. Agreement is very good: the dominant source term in this flow is the shear-noise term. Thus, although the key hydrodynamic fluctuations are generated through a nonlinear mechanism, the source of sound itself is generated linearly. This result is significant since all approaches based on TABF decompositions associate the linear terms with sound propagation rather than sound generation.

\section{CONCLUSION}

The silent base flow sources have been computed and validated successfully for a Mach 0.9 axisymmetric jet, at three different frequencies. The SBF sources have two interesting properties: their order of magnitude is similar to that of the sound field, and they are maximum near the end of the potential core. Moreover, the SBF sources appear to generate little to no hydrodynamic waves when used as source terms on the right hand side of the linearised Euler equations. These findings suggest that the SBF sources are close to the true sources of sound.

Sources based on a time-averaged base flow (TABF), and on a quiescent base flow (QBF) have also been computed and validated. The amplitude and distribution of the SBF sources are more physical than those of the TABF and QBF sources. The major drawback of the TABF and QBF sources is that apart from generating sound, they generate large vorticity waves which originate from the hydrodynamic components of these sources. These hydrodynamic components dominate the core radiating components, which become inaccessible. The SBF sources avoid this problem and therefore offer a critical improvement over classical acoustic analogies.

The algorithm proposed for computing the radiating part of a flow field is useful to assess the validity of a source model. If the model captures the dominant source mechanism, then its radiating part should be a good approximation to the SBF sources. Thus, we show that in the laminar jet presented in this paper, the dominant noise mechanism is the "shear-noise" term. It involves a product of the mean flow with the hydrodynamic fluctuations, along the jet axis. The source takes the form of a wavepacket whose envelope is nonuniform. At this stage, it is unclear whether such mechanism plays a significant role in high speed turbulent jets. This issue will be settled by applying the method presented here to a turbulent jet simulated numerically.

\section{ACKNOWLEDGMENTS}

This project was funded by the Engineering and Physical Sciences Research Council under the Ph.D. Plus programme. The authors would like to thank Dr. Victoria Suponitsky and Professor Neil Sandham, who carried out the DNS simulation used in this paper, Dr. Ed Brambley, for suggesting to compute the radiating part of the QBF sources and Dr. Alexandre Kabla for editorial advice.

\section{APPENDIX A: GOVERNING EQUATIONS}

\section{Silent base flow}

Using the silent base flow decomposition, the governing equations can be written as

$$
\begin{aligned}
& \frac{\partial \rho^{\prime}}{\partial t}+\frac{\partial}{\partial x_{j}}\left(\rho v_{j}\right)^{\prime}=0, \\
& \frac{\partial}{\partial t}\left(\rho v_{i}\right)^{\prime}+\frac{\partial}{\partial x_{j}}\left[\tilde{v}_{j}\left(\rho v_{i}\right)^{\prime}+\tilde{v}_{i}\left(\rho v_{j}\right)^{\prime}-\tilde{v}_{i} \tilde{v}_{j} \rho^{\prime}\right]^{\prime} \\
& \quad+\gamma \frac{\partial}{\partial x_{i}}\left[\bar{\pi}^{\gamma-1} \pi^{\prime}\right]^{\prime}=f_{1 i}, \\
& \frac{\partial \pi^{\prime}}{\partial t}+\frac{\partial}{\partial x_{j}}\left[\frac{\bar{\pi}}{\bar{\rho}}\left[\left(\rho v_{j}\right)^{\prime}-\tilde{v}_{j} \rho^{\prime}\right]+\tilde{v}_{j} \pi^{\prime}\right]^{\prime}=0 .
\end{aligned}
$$

\section{Time-averaged base flow}

Using the time-averaged base flow decomposition, the governing equations can be written as

$$
\begin{aligned}
& \frac{\partial \rho^{\prime \prime}}{\partial t}+\frac{\partial}{\partial x_{j}}\left(\rho v_{j}\right)^{\prime \prime}=0 \\
& \frac{\partial}{\partial t}\left(\rho v_{i}\right)^{\prime \prime}+\frac{\partial}{\partial x_{j}}\left(\hat{v}_{j}\left(\rho v_{i}\right)^{\prime \prime}+\hat{v}_{i}\left(\rho v_{j}\right)^{\prime \prime}-\hat{v}_{i} \hat{v}_{j} \rho^{\prime \prime}\right) \\
& \quad+\gamma \frac{\partial}{\partial x_{i}} \pi_{0}^{\gamma-1} \pi^{\prime \prime}=f_{2 i}, \\
& \frac{\partial \pi^{\prime \prime}}{\partial t}+\frac{\partial}{\partial x_{j}}\left(\frac{\pi_{0}}{\rho_{0}}\left(\rho v_{j}\right)^{\prime \prime}+\hat{v}_{j} \pi^{\prime \prime}-\frac{\pi_{0}}{\rho_{0}} \hat{v}_{j} \rho^{\prime \prime}\right)=0
\end{aligned}
$$

\section{Quiescent base flow}

Using the quiescent base flow decomposition, the governing equations can be written as 


$$
\begin{aligned}
& \frac{\partial}{\partial t}\left(\rho-\rho_{\infty}\right)+\frac{\partial}{\partial x_{j}}\left(\rho v_{j}\right)=0 \\
& \frac{\partial}{\partial t}\left(\rho v_{i}\right)+\gamma \pi_{\infty}^{\gamma-1} \frac{\partial}{\partial x_{i}}\left(\pi-\pi_{\infty}\right)=f_{3 i}, \\
& \frac{\partial}{\partial t}\left(\pi-\pi_{\infty}\right)+\frac{\partial}{\partial x_{j}}\left(\frac{\pi_{\infty}}{\rho_{\infty}} \rho v_{j}\right)=0 .
\end{aligned}
$$

\section{APPENDIX B: SPATIAL FOURIER TRANSFORM OF AN AXISYMMETRIC FIELD IN THE RADIAL DIRECTION}

In the following, the flow fields are studied in a plane orthogonal to the jet axis, i.e., for a given value $z=z_{0}$. In this plane, we use the polar coordinate systems $(r, \theta)$ in the physical space, and the polar coordinate system $\left(k_{r}, \phi\right)$ in the wavenumber space.

Let $q(r)$ denote an axisymmetric scalar flow variable in the physical plane $z=z_{0}$. Its Fourier transform is given by

$$
\begin{aligned}
Q\left(k_{r}, \phi\right) & =\int_{0}^{+\infty} \int_{0}^{2 \pi} q(r) e^{i k_{r} r \cos (\theta-\phi)} r d \theta d r \\
& =2 \pi \int_{0}^{+\infty} q(r) J_{0}\left(k_{r} r\right) r d r
\end{aligned}
$$

which equals $2 \pi$ times the Hankel transform of the first kind of order 0 of $q$.

In the $z=z_{0}$ plane, the velocity field can be expressed as $\mathbf{v}=v_{r} \mathbf{e}_{\mathrm{r}}=v_{r} \cos \theta \mathbf{e}_{\mathbf{x}}+v_{r} \sin \theta \mathbf{e}_{\mathbf{y}}$, so its Fourier transform is given by

$$
\begin{aligned}
\mathbf{V}\left(k_{r}, \phi\right)= & \int_{0}^{+\infty} \int_{0}^{2 \pi} v_{r}(r) \cos \theta e^{i k_{r} r \cos (\theta-\phi)} r d \theta d r \mathbf{e}_{\mathbf{x}} \\
& +\int_{0}^{+\infty} \int_{0}^{2 \pi} v_{r}(r) \sin \theta e^{i k_{r} r \cos (\theta-\phi)} r d \theta d r \mathbf{e}_{\mathbf{y}} \\
= & 2 \pi i \int_{0}^{+\infty} q(r) J_{1}\left(k_{r} r\right) r d r\left(\cos \phi \mathbf{e}_{\mathbf{x}}+\sin \phi \mathbf{e}_{\mathbf{y}}\right) \\
= & 2 \pi i \int_{0}^{+\infty} q(r) J_{1}\left(k_{r} r\right) r d r \mathbf{e}_{\mathbf{r}},
\end{aligned}
$$

which equals $2 \pi i$ times the Hankel transform of the first kind of order 1 of $q$.

${ }^{1}$ M. E. Goldstein, “A generalized acoustic analogy,” J. Fluid Mech. 488, 315-333 (2003).

${ }^{2}$ M. J. Lighthill, "On sound generated aerodynamically. I. General theory," Proc. R. Soc. London 211, 564-587 (1952).

${ }^{3}$ G. M. Lilley, "On the noise from jets," in AGARD Conference Proceedings No. 131 on Noise Mechanisms, Brussels, Belgium (1974).

${ }^{4}$ C. Bogey, C. Bailly, and D. Juvé, "Computation of flow noise using source terms in linearized Euler's equations," AIAA J. 40, 235-243 (2002).

${ }^{5} \mathrm{M}$. E. Goldstein, "On identifying the true sources of aerodynamic sound," J. Fluid Mech. 526, 337-347 (2005).

${ }^{6} \mathrm{M}$. Cabana, V. Fortuné, and P. Jordan, "Identifying the radiating core of Lighthill's source term," Theor. Comput. Fluid Dyn. 22, 87-106 (2008).

${ }^{7}$ S. Sinayoko, A. Agarwal, and Z. Hu, "Flow decomposition and aerodynamic noise generation," J. Fluid Mech. 668, 335-350 (2011).

${ }^{8} \mathrm{P}$. Jordan and Y. Gervais, "Subsonic jet aeroacoustics: associating experiment, modeling and simulation," Exp. Fluids 44, 1-21 (2008).

${ }^{9}$ C. Bogey, C. Bailly, and D. Juvé, "Noise investigation of a high subsonic, moderate Reynolds number jet using a compressible large eddy simulation," Theor. Comput. Fluid Dyn. 16, 273-297 (2003).

${ }^{10}$ J. E. Ffowcs Williams and D. L. Hawkings, "Sound generation by turbulence and surfaces in arbitrary motion," Philos. Trans. R. Soc. London, Ser. A 264, 321-342 (1969).

${ }^{11}$ G. M. Lilley, "The generation and radiation of supersonic jet noise," Vol. IV of Theory of Turbulence Generated Jet Noise, Noise Radiation From Upstream Sources, and Combustion Noise. Part II: Generation of Sound in a Mixing Region, AFAPL-TR-72-53 (US Air Force Aero Propulsion Laboratory, Wright-Patterson Air Force Base, OH, 1972).

${ }^{12}$ C. K. W. Tam, "Supersonic jet noise," Annu. Rev. Fluid Mech. 27, 17-43 (1995).

${ }^{13}$ D. G. Crighton, "Basic principles of aerodynamic noise generation," Prog. Aerospace Sci. 16, 31-96 (1975).

${ }^{14} \mathrm{G}$. Goertzel, "An algorithm for the evaluation of finite trigonometric series," Am. Math. Monthly 65, 34-35 (1958).

${ }^{15}$ J. L. Stromberg, D. K. McLaughlin, and T. R. Troutt, "Flow field and acoustic properties of a Mach number 0.9 jet at a low Reynolds number," J. Sound Vibr. 72, 159-176 (1980).

${ }^{16}$ V. Suponitsky and N. D. Sandham, "Nonlinear mechanisms of sound radiation in a subsonic flow," AIAA paper 2009-3317, 2009.

${ }^{17}$ V. Suponitsky, N. D. Sandham, and C. L. Morfey, "Linear and nonlinear mechanisms of sound radiation by instability waves in subsonic jets," J. Fluid Mech. 658, 509-538 (2010).

${ }^{18}$ J. Panda and R. G. Seasholtz, "Experimental investigation of density fluctuations in high-speed jets and correlation with generated noise," J. Fluid Mech. 450, 97-130 (2002).

${ }^{19}$ J. Panda, R. G. Seasholtz, and K. A. Elam, "Investigation of noise sources in high-speed jets via correlation measurements," J. Fluid Mech. 537, 349-385 (2005). 